\title{
Cause-of-Death Contributions to Educational Inequalities in Mortality in Austria between 1981/1982 and 1991/1992
}

\section{Les contributions des causes de décès aux inégalités de mortalité par niveau d'éducation en Autriche entre 1981/1982 et 1991/1992}

\section{Roland Rau • Gabriele Doblhammer • Vladimir Canudas-Romo $\cdot$ Zhang Zhen}

Received: 21 February 2007/ Accepted: 21 October 2007/Published online: 21 November 2007 (C) Springer Science+Business Media B.V. 2007

\begin{abstract}
This article uses census records and deaths records to analyze trends in educational inequalities in mortality for Austrian women and men aged 35-64 years between 1981/1982 and 1991/1992. We find an increasing gradient in mortality by education for circulatory diseases and especially ischaemic heart disease. Respiratory diseases and, in addition for women, cancers showed the opposite trend. Using decomposition analysis, we give evidence that in many cases changes in the agestructure within the 10-year interval had a bigger effect than direct improvements in mortality on the analyzed subpopulations.
\end{abstract}

Keywords Austria - Causes of death - Decomposition analysis ·

Education · Mortality · Trends · Socioeconomic factors

Résumé Cet article utilise les données de recensement et certificats de décès pour analyser les tendances en matière d'inégalités de mortalité par niveau d'éducation pour les Autrichiens des deux sexes âgés de 35 à 64 ans entre 1981/1982 et 1991/ 1992. Le gradient de mortalité par niveau d'éducation a tendance à s'accentuer pour les maladies circulatoires et particulièrement pour les maladies ischémiques. La tendance opposée apparaît pour les maladies respiratoires, et, chez les femmes

\footnotetext{
R. Rau $(\bowtie)$

Terry Sanford Institute of Public Policy, Population, Policy, and Aging Research Center (PPARC), Duke University, Box 90309, 302 Towerview Road, Durham, NC 27708-0309, USA

e-mail: roland.rau@duke.edu

G. Doblhammer

University of Rostock, Rostock, Germany

V. Canudas-Romo

Johns Hopkins Bloomberg School of Public Health, Baltimore, MD, USA
}

Z. Zhen

Max Planck Institute for Demographic Research, Rostock, Germany 
seulement, pour les cancers. En utilisant l'analyse par décomposition, nous démonstrons que des changements de structure par âge au cours de la période de 10 ans peuvent avoir un impact plus important sur les inégalités par niveau d'éducation que les baisses de mortalité.

Mots-clés Autriche - Causes de décès - Analyse par décomposition · Éducation · Mortalité · Tendances · Facteurs socio-économiques

\section{Introduction}

Socioeconomic mortality differentials in Austria increased between the 1980s and 1990s for people at working ages (35-64 years) as two papers have recently shown (Doblhammer et al. 2005; Schwarz 2005). This unfavorable development applied mainly to men: the relative mortality risk of men with tertiary education was $43 \%$ lower in relation to men with basic education in 1981/1982. Ten years later, the relative mortality risk was $54 \%$ lower (Doblhammer et al. 2005). A similar, but less dramatic, development has been observed for women, where the corresponding risks increased from 20 to 26\%. Austria does not represent an exception with this development. Growing socioeconomic mortality disparities have been observed for various European countries as well as for the United States during recent decades, typically with stronger effects for men than for women (e.g., Davey Smith et al. 1990; Feldman et al. 1989; Kunst et al. 2004; Lauderdale 2001; Leinsalu et al. 2003; Mackenbach et al. 2003; Martikainen et al. 2001; Pappas et al. 1993; Preston and Elo 1995; Valkonen 1999, 2006). What sets Austria apart from most of the other studies is an increase not only in relative, but also in absolute mortality levels at adult ages for men.

The aim of this article is to assess which causes of death have been crucial for the described widening gap in socioeconomic mortality differentials among women and men in Austria at ages 35-64 ("working ages"). The motivation stems from the question whether there is "a common background behind growing inequalities in mortality in Western European countries?" (Koskinen 2003, p. 838). The literature suggests that diseases of the circulatory system play an important role. By reviewing numerous studies, Valkonen (1999) concludes "the decline in cardiovascular mortality has been more rapid in the upper than in the lower socio-economic men in all countries for which we have data. This difference in trends has been the most important single cause for the widening of the socioeconomic mortality gap" (p. 309). Other causes contributed as well, but their impact is hard to generalize (Koskinen 2003; Valkonen 1999). In a more recent study for Finland, Norway, England and Wales, Belgium, Austria, Switzerland, Turin, and Barcelona and Madrid, Huisman et al. (2005a) obtain similar results with respect to the role of circulatory diseases: "Cardiovascular diseases contributed the most to these differences in mortality. In men, the top five specific contributory causes were ischaemic heart disease, lung cancer, COPD, other cardiovascular diseases, and 
cerebrovascular diseases. In women they were ischaemic heart disease, other cardiovascular diseases, cerebrovascular diseases, pneumonia, and COPD" (p. 497). ${ }^{1}$ Hence, our aim is to "go beyond the simple assessment such as the gap is widening", as suggested by Kunst et al. (2004, p. 231) and analyze the impact of various causes of death on women and men at working ages. Due to their prominent position in the literature, cardiovascular and cerebrovascular diseases are analyzed in more detail than other causes.

\section{Data and Methods}

\subsection{Data}

\subsubsection{Merging of Data}

Our data are constructed by linking the census records from 1981 to 1991 with the subsequent death records for 1 year: 12 May 1981 to 11 May 1982 and 15 May 1991 to 14 May 1992. Mortality data and information on socioeconomic characteristics on the individual level for the whole population are only available for those two time periods in Austria. Since Austria had at that time no population register with unique personal identification numbers, the linkage was made using the following variables: birth date (day, month, year), marital status (if married, also year of marriage) and last residential address. Out of the 90,693 death certificates submitted to the central bureau of statistics in 1981/1982, 1,389 records (1.53\%) had to be excluded due to erroneous dwelling information. Out of the remaining 89,304 certificates, $91.62 \%$ could be linked to the census records. With a proportion of $90.09 \%$ in 1991/1992, the merging rate was only slightly less successful. This lower value can probably be explained by the absence of the variable 'marital status' in the 1991/1992 linkage procedure. Residential mobility between the census date and the (potential) date of death is the main factor to determine whether a death record can be linked successfully to a census record. We made some comparisons of the distribution of characteristics in (a) all death records and (b) the linked death records. Linkage success is not uniformly distributed across age: in our age-range (35-64 years), the merging rate is even higher than in the population as a whole. This is caused by the fact that younger people as well as old people are more likely to change residence (e.g., leaving home for the young, and entering a nursing home for the elderly). We discovered that the merging rate for women was lower than for men. Due to the higher life expectancy of women, men are more likely to be cared for at home than women. Geographically, the merging differed between the states (Länder) of Austria. In Burgenland - a rural region, where the highest linkage rate was recorded-elderly individuals tend to live with their relatives, whereas in Vienna, the most urban of the nine Länder, merging success was lower. Further details of the linkage, such as the scoring algorithm for matching death and census records, are explained in Doblhammer (1997) and Doblhammer et al. (2005).

\footnotetext{
${ }^{1}$ COPD is the abbreviation for "chronic obstructive pulmonary disease".
} 


\subsubsection{Choice of Education as Indicator for Socioeconomic Status}

Regardless of whether socioeconomic status (SES) is measured via income, wealth, education, or occupation, an inverse relationship with mortality is found (Goldman 2001). Mortality is typically lower with higher income, more wealth, and more time spent in education (e.g., Kitagawa and Hauser 1973; Kunst 1997). The choice of indicator does not only reflect an academic tradition where, for example, British researchers tend to focus on occupational groups and American researchers on education to measure socioeconomic status (Christenson and Johnson 1995; Davey Smith et al. 1998). Each of these indicators addresses also a different health dimension: whereas the kind of job might be more related to occupational hazards, income could affect housing conditions and access to medical care. Despite the conclusion of Davey Smith et al. (1998, p. 153) that "as a single indicator of socioeconomic position occupational social class is a better discriminator of socioeconomic differences in mortality [...] than is education", we chose, nevertheless, to use education as an indicator for socioeconomic status in our analysis for several theoretical and practical reasons (see, for example: Elo et al. 2006; Liberatos et al. 1988; Valkonen 1989, 1999). Most importantly, educational level remains fairly stable throughout adult life. It applies equally to women and men, irrespective of whether they are in the active labor force or not. In addition, a decrease in health may affect occupational status but not educational level. ${ }^{2}$ Since education can be expected to address rather the behavioral and lifestyle dimension of health (Lynch et al. 1997), ${ }^{3}$ it can be considered a good proxy variable for risk factors that have been singled out by Marmot (2005) to be important for socioeconomic mortality differentials: obesity, smoking, alcohol, and poor diet. There are, however, also practical reasons to focus on education rather than occupation. First, the previous study by Doblhammer et al. (2005) has shown that the trends by education are clearer than by occupation. Second, the changes between 1981 and 1991 in absolute numbers by occupation as given in Doblhammer et al. (2005, pp. 472-473) suggest a change in the classification of occupations between the two census dates.

The Austrian census used seven categories for education which were grouped into three categories: "Allgemeinbildende Pflichtschule" was used as "Low" education, women and men with "Hochschule" and "Hochschulverwandte Ausbildung" were considered to have "High" education. The remaining groups ("Lehre", "Fachschule", "Allgemeinbildende Höhere Schule" and "Berufsbildende Höhere Schule") were put into the category "Medium". Table 1 shows the

\footnotetext{
${ }^{2}$ It should be mentioned that in recent years the direction of causation has been discussed with medical scientists being "often convinced that the dominant if not exclusive pathway is that variation in socioeconomic status produces health disparities. [...] Economists are now making contributions about the alternative pathway" (Smith 1999, p. 145).

3 As Smith (2005, p. 21) puts it when discussing the impact of education: "One possibility is that the education experience itself has little to do with it, but it is simply a marker for personal traits [...] that may lead people to acquire more education and to be healthier.
} 
distributions of women and men aged 35-64 by educational level and survival status for both periods of analysis. All percentages sum up to 100 column-wise. For each subpopulation, between 1.2 (Men 1981/1982: 1,222,873) and 1.4 million individuals (Women 1991/1992: 1,412,312) were exposed to the risk of dying. Slightly less than 10,000 men died during each of the two periods, whereas 5,532 women died in $1981 / 1982$ and 4,775 10 years later. In both subpopulations during both time periods, people with "high" education constitute the smallest group. "Low" education is the predominant group among women; the most common group among men is "medium" education.

By contrasting "high" and "low" education in the remainder of this article, we deliberately leave out large parts of the population ("medium education"). Since the socio-economic gradient in mortality in Austria is monotonous along the educational categories, as shown previously by Doblhammer et al. (2005), this does not affect the main goal of our article: to analyze the full extent of socioeconomic inequality in mortality in Austria over time and the role various causes of death contribute to this development.

Table 1 Numbers of women and men aged 35-64 by educational level at census date and survival status 1 year after census

\begin{tabular}{|c|c|c|c|c|c|c|}
\hline \multirow[t]{2}{*}{ Education } & \multicolumn{2}{|l|}{ Survived } & \multicolumn{2}{|l|}{ Died } & \multicolumn{2}{|l|}{ All } \\
\hline & Counts & $\%$ & Counts & $\%$ & Counts & $\%$ \\
\hline \multicolumn{7}{|c|}{ Women, 1981/1982 } \\
\hline Low & 849,861 & 62.55 & 3,931 & 71.06 & 853,792 & 62.59 \\
\hline Medium & 482,194 & 35.49 & 1,533 & 27.71 & 483,727 & 35.46 \\
\hline High & 26,567 & 1.96 & 68 & 1.23 & 26,635 & 1.95 \\
\hline$\sum$ & $1,358,622$ & 100.00 & 5,532 & 100.00 & $1,364,154$ & 100.00 \\
\hline \multicolumn{7}{|c|}{ Women, 1991/1992 } \\
\hline Low & 698,646 & 49.64 & 2,945 & 61.68 & 701,591 & 49.68 \\
\hline Medium & 645,937 & 45.89 & 1,726 & 36.15 & 647,663 & 45.86 \\
\hline High & 62,954 & 4.47 & 104 & 2.18 & 63,058 & 4.46 \\
\hline$\sum$ & $1,407,537$ & 100.00 & 4,775 & 100.00 & $1,412,312$ & 100.00 \\
\hline \multicolumn{7}{|c|}{ Men, 1981/1982 } \\
\hline Low & 459,254 & 37.85 & 4,607 & 47.85 & 463,861 & 37.93 \\
\hline Medium & 684,568 & 56.42 & 4,726 & 49.08 & 689,294 & 56.37 \\
\hline High & 69,422 & 5.72 & 296 & 3.07 & 69,718 & 5.70 \\
\hline$\sum$ & $1,213,244$ & 100.00 & 9,629 & 100.00 & $1,222,873$ & 100.00 \\
\hline \multicolumn{7}{|c|}{ Men, 1991/1992 } \\
\hline Low & 390,652 & 28.39 & 3,797 & 40.50 & 394,449 & 28.47 \\
\hline Medium & 878,940 & 63.87 & 5,308 & 56.61 & 884,248 & 63.83 \\
\hline High & 106,455 & 7.74 & 271 & 2.89 & 106,726 & 7.70 \\
\hline$\sum$ & $1,376,047$ & 100.00 & 9,376 & 100.00 & $1,385,423$ & 100.00 \\
\hline
\end{tabular}




\subsubsection{Causes of Death}

Causes of death were coded in the death certificates in 1981/1982 as well as in 1991/ 1992 using the ninth revision of the International Classification of Diseases (ICD-9). Therefore we did not have the typical problems which emerge when reconstructing causes of death across various ICD revisions (see, for example, Meslé and Vallin 1996). We encountered, however, problems which can be possibly attributed to changes in coding practice-a phenomenon that is not uncommon when working with causes of death (see, for example, Shkolnikov et al. 1996): 1,111 deaths among men at ages 35-64 were attributed to diseases of the digestive system in 1981/1982, among them 325 were due to liver cirrhosis (30\%) and 786 due to other digestive diseases $(70 \%)$. Ten years later, 1,178 men died of digestive diseases where roughly $81 \%$ were counted as liver cirrhosis (959 deaths) and only 19\% (219 deaths) as caused by other digestive diseases.

In order to avoid these problems in conjunction with the relatively low number of deaths in the age-range we are analyzing, we used large groups for causes of death apart from circulatory diseases which we grouped into "Ischaemic Heart Disease", "Cerebrovascular Diseases," and "Other Circulatory Diseases". Table 2 gives an overview of the causes of death we analyzed, what their respective ICD-9 codes are and how they were distributed between women and men aged 35-64 during the two analyzed time periods in Austria. The most common cause of death among women was cancer $(43.75 \%)$, men died most often from all kinds of circulatory diseases, especially from ischaemic heart disease (20.50\%). The residual category "Rest" consists mainly of external causes of death such as accidents. However, other causes of death can be found in that category (e.g., infectious diseases); the latter occur, however, so rarely, that they cannot constitute a category of their own.

\subsection{Methods}

We analyze the changes in socioeconomic mortality differentials using two different methods: relative mortality differentials and absolute mortality differentials. Relative differences are typically easy to comprehend; nevertheless, it is important to take into account absolute measurements for mortality since a " $50 \%$ higher rate of a rare health problem may be much less important than a $10 \%$ higher rate of a frequent health problem" (Mackenbach and Kunst 1997, p. 759). Using both approaches_relative and absolute mortality measurements-might also help to bridge the gap which appears to exist between a public health policy perspective interested mainly in absolute mortality on the one hand, and the practice of epidemiologists on the other hand who typically work with relative mortality measurements (Valkonen 1999).

Relative mortality differentials were estimated using a Generalized Linear Model (GLM) framework for binary data since the response we are interested in is the state of an individual at the end of the observation period, i.e., either alive or dead. We 
Table 2 Numbers of death by cause of death for women and men for time periods 1981/1982 and 1991/ 1992 (absolute and relative frequencies)

\begin{tabular}{|c|c|c|c|c|c|c|c|}
\hline \multirow[t]{2}{*}{ Cause of death } & \multirow[t]{2}{*}{ ICD-9 Code } & \multicolumn{2}{|c|}{ 1981/1982 } & \multicolumn{2}{|c|}{ 1991/1992 } & \multicolumn{2}{|l|}{ Total } \\
\hline & & Counts & $\%$ & Counts & $\%$ & Counts & $\%$ \\
\hline \multicolumn{8}{|l|}{ Women } \\
\hline Cancers & $140-239$ & 2,307 & 41.70 & 2,203 & 46.14 & 4,510 & 43.76 \\
\hline Ischaemic heart disease & $410-414$ & 585 & 10.57 & 558 & 11.69 & 1,143 & 11.09 \\
\hline Cerebrovascular diseases & $430-438$ & 440 & 7.95 & 410 & 8.59 & 850 & 8.25 \\
\hline All other circul. diseases & $390-459$ & 625 & 11.30 & 427 & 8.94 & 1,052 & 10.21 \\
\hline Respiratory diseases & $460-519$ & 147 & 2.66 & 117 & 2.45 & 264 & 2.56 \\
\hline Diseases of the digestive system & $520-579$ & 419 & 7.57 & 373 & 7.81 & 792 & 7.68 \\
\hline Rest & Remainder & 1,009 & 18.24 & 687 & 14.39 & 1,696 & 16.45 \\
\hline Total & All & 5,532 & 100.00 & 4,775 & 100.00 & 10,307 & 100.00 \\
\hline \multicolumn{8}{|l|}{ Men } \\
\hline Cancers & $140-239$ & 2,532 & 26.30 & 2,936 & 31.31 & 5,468 & 28.77 \\
\hline Ischaemic heart disease & $410-414$ & 2,061 & 21.40 & 1,835 & 19.57 & 3,896 & 20.50 \\
\hline Cerebrovascular diseases & $430-438$ & 636 & 6.61 & 707 & 7.54 & 1,343 & 7.07 \\
\hline All other circul. diseases & $390-459$ & 886 & 9.20 & 619 & 6.60 & 1,505 & 7.92 \\
\hline Respiratory diseases & $460-519$ & 313 & 3.25 & 290 & 3.09 & 603 & 3.17 \\
\hline Diseases of the digestive system & $520-579$ & 1,111 & 11.54 & 1,178 & 12.56 & 2,289 & 12.04 \\
\hline Rest & Remainder & 2,090 & 21.71 & 1,811 & 19.32 & 3,901 & 20.53 \\
\hline Total & All & 9,629 & 100.00 & 9,376 & 100.00 & 19,005 & 100.00 \\
\hline \multicolumn{8}{|l|}{ Total (Women and Men) } \\
\hline Cancers & $140-239$ & 4,839 & 31.92 & 5,139 & 36.32 & 9,978 & 34.04 \\
\hline Ischaemic heart disease & $410-414$ & 2,646 & 17.45 & 2,393 & 16.91 & 5,039 & 17.19 \\
\hline Cerebrovascular diseases & $430-438$ & 1,076 & 7.10 & 1,117 & 7.89 & 2,193 & 7.48 \\
\hline All other circul. diseases & $390-459$ & 1,511 & 9.97 & 1,046 & 7.39 & 2,557 & 8.72 \\
\hline Respiratory diseases & $460-519$ & 460 & 3.03 & 407 & 2.88 & 867 & 2.96 \\
\hline Diseases of the digestive system & $520-579$ & 1,530 & 10.09 & 1,551 & 10.96 & 3,081 & 10.51 \\
\hline Rest & Remainder & 3,099 & 20.44 & 2,498 & 17.65 & 5,597 & 19.09 \\
\hline Total & All & 15,161 & 100.00 & 14,151 & 100.00 & 29,312 & 100.00 \\
\hline
\end{tabular}

chose the canonical link for binary data, the logistic link. The model we estimated was

$$
\log \left(\frac{\pi_{i}}{1-\pi_{i}}\right)=\alpha+\sum_{\text {age }=40-44 y}^{60-64 y} \beta_{\text {age }} x_{\text {age }, i}+\sum_{\text {edu }=\text { Medium }}^{\text {High }} \gamma_{\text {edu }} x_{\text {edu }, i}
$$

where the probability of dying of individual $i$ is denoted as $\pi_{i}$; the estimated parameters are the intercept $\alpha$, five $\beta$ parameters to control for age (each age-group spans 5 years with ages 35-39 employed as the reference category) and two $\gamma$ parameters for the effect of medium and high education (low education served as the baseline category). We did not control for any other covariates apart from age 
since we wanted to be able to compare our results from both approaches (relative and absolute mortality differentials). One of the major advantages of using a logit link (instead of the probit or the complementary $\log -\log$, for instance) is the interpretation of the estimated parameters (McCullagh and Nelder 1989). Exponentiating the parameter estimates allows to interpret odds ratios as relative risks if the number of events is relatively small in comparison to the number of exposures (Woodward 1999), a condition which is fulfilled for our analysis.

Absolute mortality differentials were analyzed using Decomposition Analysis. Decomposition of mortality rates can be considered to be the other side of the coin of the typical approach used to analyze absolute mortality differentials via agestandardized mortality rates (Das Gupta 1994). Instead of comparing artificial rates (due to standardization with an arbitrary population), the focus is on the observed rates and their changes over time (or other categories). Decomposition analysis in general enables to disentangle the "components of a difference of two rates" (Kitagawa 1955). The difference between two observed rates can be split up into a direct component (e.g., change in mortality) and a compositional component (e.g., change in age structure). Our focus of interest is, however, not a simple decomposition of two rates but a "difference of differences":

- The differences in mortality between 1981/1982 and 1991/1992 for education "High" minus;

- The differences in mortality between 1981/1982 and 1991/1992 for education "Low".

Our decomposition analysis closely follows the notation and methods outlined in Vaupel and Canudas-Romo (2002, 2003), Vaupel et al. (2006) and Canudas-Romo (2003). In the remainder of this section, we will use the following notation: age is denoted by $a, e_{j}$ represents the two educational levels of interest: high $(j=1)$ and low $(j=2)$ education. The two time periods 1981/1982 and 1991/1992 are expressed as $t_{k}, k=1,2$; subscript $i$ denotes the $i$ th cause of death (CoD). The agespecific size of a population at age $a$ with $e_{j}$ at time $t_{k}$ is $N\left(a, e_{j}, t_{k}\right)$ and the covariance between death rates $d_{i}$ and growth rates of the proportions of age-groups $N$ is represented by $\operatorname{Cov}\left(d_{i}, N\right)$.

With this notation we can write the death rate of a population with $e_{j}$ at time $t_{k}$ as

$$
d\left(e_{j}, t_{k}\right)=\sum_{i} \bar{d}_{i}\left(e_{j}, t_{k}\right)
$$

where the death rate of people with $e_{j}$ who died of the $i$ th CoD at time $t_{k}, \bar{d}_{i}\left(e_{j}, t_{k}\right)$, is actually the average of age-education-time-specific death rates for the $i$ th CoD, that is

$$
\bar{d}_{i}\left(e_{j}, t_{k}\right)=\frac{\sum_{a} d_{i}\left(a, e_{j}, t_{k}\right) N\left(a, e_{j}, t_{k}\right)}{\sum_{a} N\left(a, e_{j}, t_{k}\right)} .
$$

With (2) and (3), and assuming the independence of causes of death, we can write the death rate of a population with educational level $j$ at time $t$. For example, the death rate of a population with educational level $1\left(e_{1}\right)$ at time $t_{2}$ will be given by 


$$
\begin{aligned}
d\left(e_{1}, t_{2}\right) & =\sum_{i}\left[\frac{\sum_{a} d_{i}\left(a, e_{1}, t_{2}\right) N\left(a, e_{1}, t_{2}\right)}{\sum_{a} N\left(a, e_{1}, t_{2}\right)}\right] \\
& =\sum_{i} \bar{d}_{i}\left(e_{1}, t_{2}\right) .
\end{aligned}
$$

Now, we decompose the difference of differences in death rates between different educational levels over time. First, the difference of death rates between different educational levels at time $t_{k}$ is

$$
\Delta d\left(t_{k}\right)=d\left(e_{1}, t_{k}\right)-d\left(e_{2}, t_{k}\right)
$$

Hence, the difference of differences in death rates is

$$
\Delta d\left(t_{1}\right)-\Delta d\left(t_{2}\right)=\left[d\left(e_{1}, t_{1}\right)-d\left(e_{2}, t_{1}\right)\right]-\left[d\left(e_{1}, t_{2}\right)-d\left(e_{2}, t_{2}\right)\right] .
$$

Using the formula for decomposition of averages (see Canudas-Romo 2003; Vaupel and Canudas-Romo 2002, for example) we can decompose the change in an average $\dot{\bar{d}}_{i}$ into a direct component, denoted by the average change $\overline{\dot{d}}_{i}$, plus the covariance between the rates of interest $d_{i}$ and the growth rates of the proportions of the respective age-groups $N$, denoted by $\operatorname{Cov}\left(d_{i}, N\right)$. This allows us to write the right hand side of Eq. 6 as follows

$$
\begin{aligned}
\Delta d\left(t_{1}\right)-\Delta d\left(t_{2}\right)= & {\left[d\left(e_{1}, t_{1}\right)-d\left(e_{1}, t_{2}\right)\right]-\left[d\left(e_{2}, t_{1}\right)-d\left(e_{2}, t_{2}\right)\right] } \\
= & \sum_{i}\left[\bar{d}_{i}\left(e_{1}, t_{1}\right)-\bar{d}_{i}\left(e_{1}, t_{2}\right)\right]-\sum_{i}\left[\bar{d}_{i}\left(e_{2}, t_{1}\right)-\bar{d}_{i}\left(e_{2}, t_{2}\right)\right] \\
= & \sum_{i}\left[\bar{d}_{i}\left(e_{1}\right)-\overline{\dot{d}}_{i}\left(e_{2}\right)\right] \\
& \quad+\sum_{i}\left[\operatorname{Cov}\left(d_{i}\left(e_{1}\right), N\left(e_{1}\right)\right)-\operatorname{Cov}\left(d_{i}\left(e_{2}\right), N\left(e_{2}\right)\right)\right] .
\end{aligned}
$$

The first term in the last two lines of Eq. 7 is the direct component of the decomposition and represents the contribution of survival improvement to the difference of differences of the death rates. The second term is the contribution of compositional changes over time on the difference of differences of the death rates.

\section{Results}

\subsection{Trends in Relative Inequality}

Figure 1 shows the odds-ratios for men with high education in relation to the reference category low education including the respective $95 \%$ confidence intervals. The bar in light gray indicates results for 1981/1982 whereas darker gray indicates results for 1991/1992. The upper left panel in Fig. 1 iterates the previously described result (Doblhammer et al. 2005; Schwarz 2005): the gap in educational inequalities increased between 1981/1982 and 1991/1992. The relative risk of dying for men with high education was $48 \%$ in $1981 / 1982$. Ten years later, it dropped to $36 \%$. The remaining seven panels display for our selected causes of death whether 

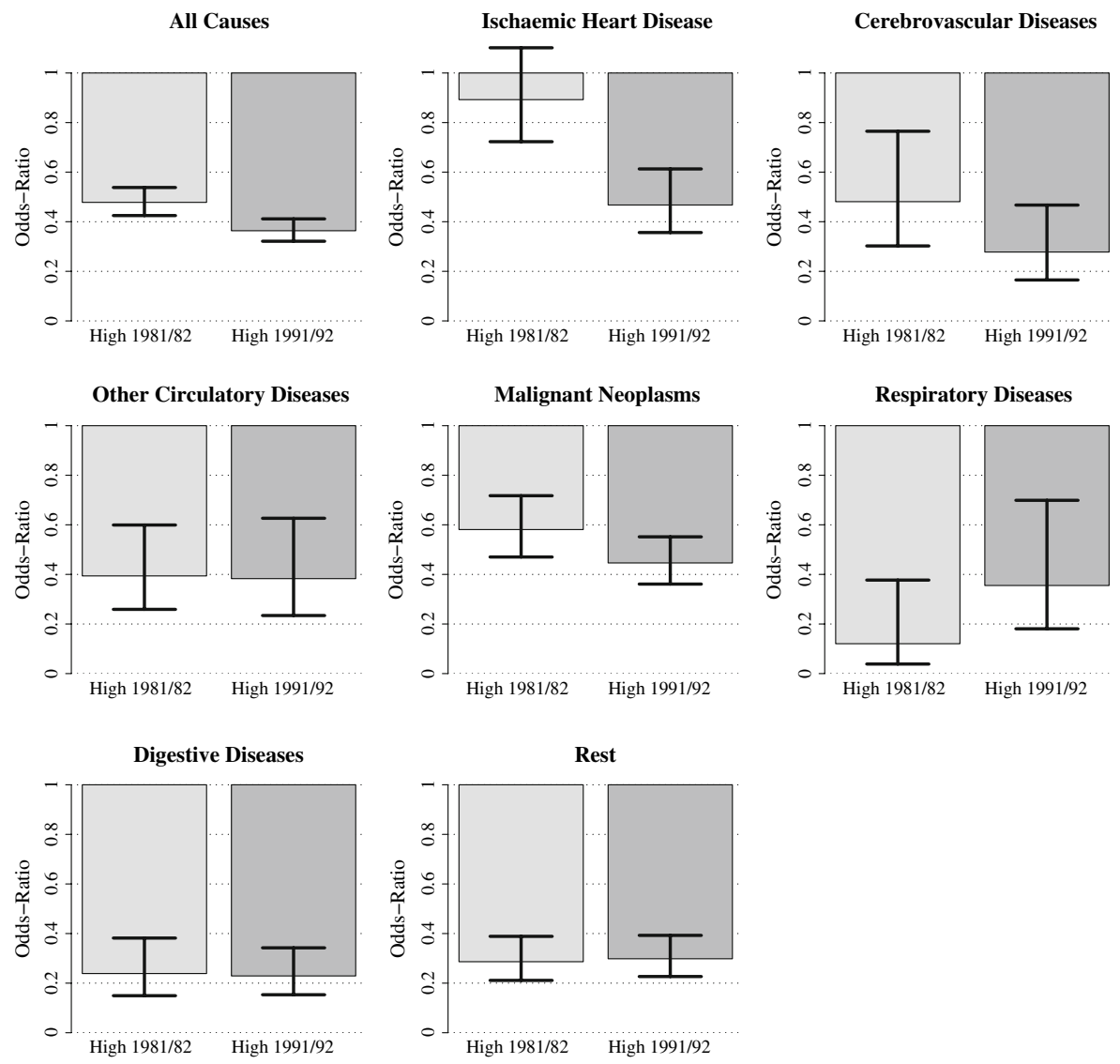

Fig. 1 Relative mortality differentials: odds-ratios and their $95 \%$ confidence intervals for men with high education in relation to the reference category low education by cause of death in 1981/1982 and 1991/ 1992

they could be relevant for this development or not. One can see that the gap in educational inequalities stagnates between the two time points for "Other Circulatory Diseases", "Digestive Diseases", and "Rest". If respiratory diseases were the major cause of death for men in Austria at those ages, the gap in all-cause mortality would have decreased. Although the gap is still considerable in 1991/1992 $(36 \%)$, the relative risk of dying from these diseases was much lower for men with high education 10 years earlier (12\%). Besides cancers and cerebrovascular diseases, the largest increase in mortality differentials by education can be attributed to ischaemic heart disease. The gradient by education was relatively flat in 1981/1982 for this disease. Within 10 years the relative mortality risk for men with high education in relation to low education fell from $89 \%$ to $47 \%$.

Previous analyses have shown that the development of mortality differentials by education are less pronounced for women than for men (Doblhammer et al. 2005; Schwarz 2005). Indeed, as our analysis shows in Fig. 2, the existing gap remained 

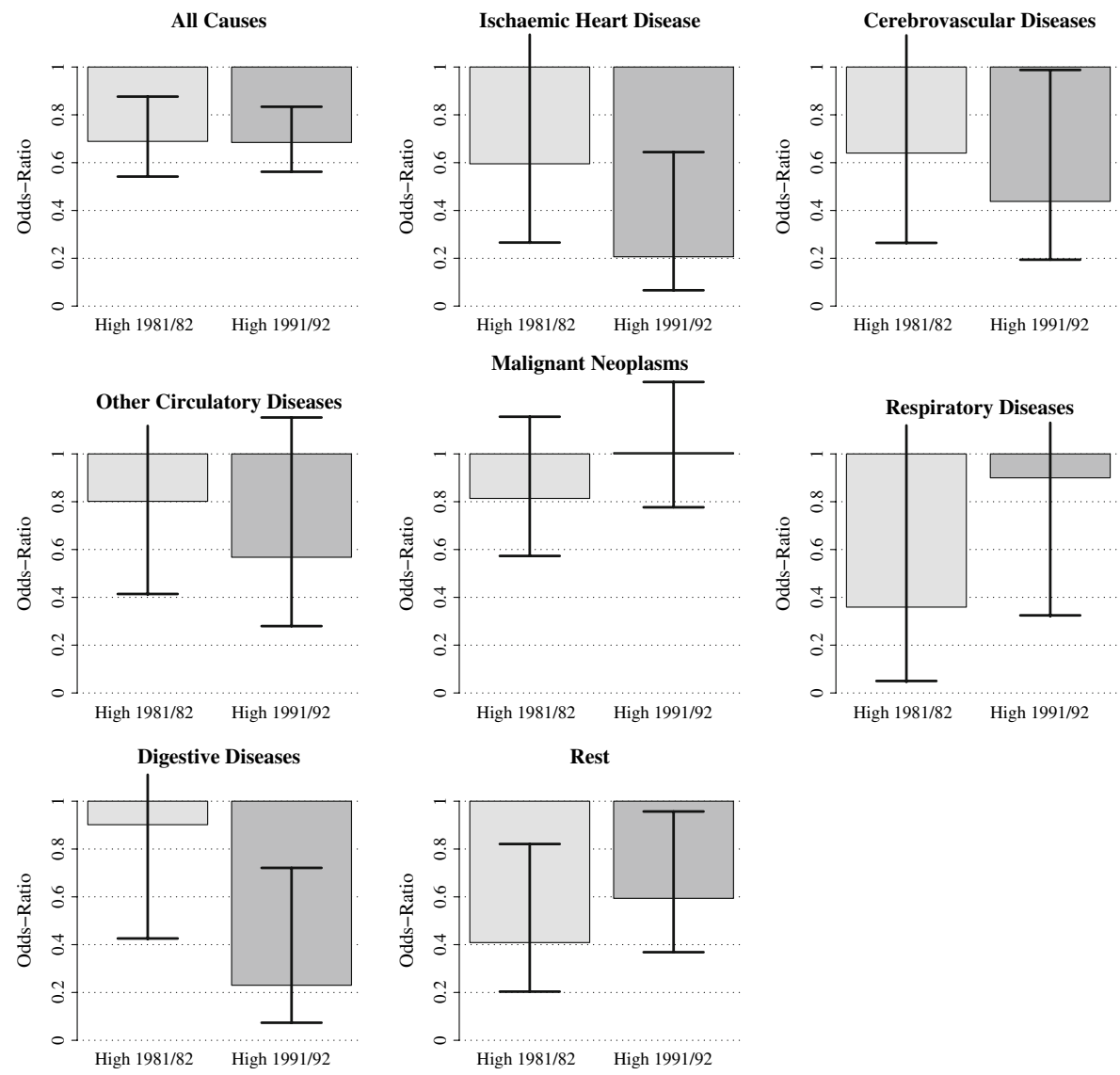

Fig. 2 Relative mortality differentials: odds-ratios and their 95\% confidence intervals for women with high education in relation to the reference category low education by cause of death in 1981/1982 and $1991 / 1992$

almost perfectly constant. ${ }^{4}$ If this pattern is broken down by cause of death, an interesting feature emerges: the stagnation in mortality differentials by education over time is the outcome of some causes of death with an increasing gap and some causes of death with a decreasing gap. Previously, existing gaps disappeared almost completely for cancers and respiratory diseases. Also the residual category would decrease the gap. Their impact is offset by all circulatory diseases (ischaemic heart disease, cerebrovascular diseases, "other circulatory diseases") and diseases of the digestive system. Relative mortality risks for women with high education in relation

\footnotetext{
${ }^{4}$ The parameter estimates are -0.372 for $1981 / 1982$ and -0.379 for $1991 / 1992$.
} 
to low education decreased between $1981 / 1982$ and $1991 / 1992$ from $60 \%$ to $21 \%$ for ischaemic heart disease, from $64 \%$ to $44 \%$ for cerebrovascular diseases and from $80 \%$ to $57 \%$ for all remaining circulatory diseases.

Summarizing the results for relative mortality differentials, we detected, for women as well as for men, an increase in mortality inequalities by education for ischaemic heart disease and cerebrovascular diseases. Respiratory diseases showed the opposite effect for both sexes whereas existing educational differentials for cancers disappeared for women. The analysis of relative mortality differentials does not indicate how relevant a certain cause of death is for all-cause mortality. This feature is taken explicitly into account when absolute mortality differentials are studied.

\subsection{Decomposition of Trends in Absolute Inequality}

Tables 3 and 4 present the results of our decomposition of absolute mortality levels. Each of these two tables is divided into three sub-tables. The top table displays the analysis for individuals with high education, the corresponding table in the middle for people with low education and the bottom table shows the difference between "high" and "low" education. In each subtable we give for each cause of death (and the sum of all causes) by columns:

- The observed death rates in 1981/1982 (or the difference between "high" and "low") and

- the observed death rates in 1991/1992 (or the difference between "high" and "low") and

- the observed difference between the previous two columns, indicated by $\Delta$, which is equivalent to $\Delta d_{i}\left(t_{1}\right)-\Delta d_{i}\left(t_{2}\right)$ following the notation of Eq. $\underline{7}$ and

- the change in mortality, denoted as "Direct", which is equivalent to $\dot{d}_{i}\left(e_{1}\right)-$ $\bar{d}_{i}\left(e_{2}\right)$ in Eq. 7 and

- the compositional component, denoted as "Comp.," which is equivalent to $\operatorname{Cov}\left(d_{i}\left(e_{1}\right), N\left(e_{1}\right)\right)-\operatorname{Cov}\left(d_{i}\left(e_{1}\right), N\left(e_{2}\right)\right)$ in Eq. 7.

Between 1981/1982 and 1991/1992 death rates for all causes combined were decreasing for men with high education and with low education (Table 3). The observed death rates (per 10,000) for men with high education dropped from 42.639 to 25.457 (-17.181). If the age-structure had been the same in 1991/1992 as in $1981 / 1982$, mortality would have dropped by 13 deaths per 10,000 (12.929). Since the compositional component points in the same direction $(-4.252)$, we can conclude that the change in the age-structure favored decreasing mortality. Typically, this is the effect of a rejuvenating population. ${ }^{5}$ The strongest direct reductions in mortality were found for men with high education for ischaemic heart

\footnotetext{
5 This is indeed the case. Assuming that the average age in each 5-year age-group is the midpoint of the respective interval, we calculated that the mean age for men with high education was 47.05 years in 1981/ 1982 and 46.15 years in 1991/1992.
} 
Table 3 Decomposition of death rates (per 10,000) of men into direct and compositional ("Comp.") components

\begin{tabular}{|c|c|c|c|c|c|}
\hline Cause of death & $\begin{array}{l}\text { Death rate } \\
1981 / 1982\end{array}$ & $\begin{array}{l}\text { Death rate } \\
1991 / 1992\end{array}$ & $\Delta$ & Direct & Comp. \\
\hline \multicolumn{6}{|l|}{ High education } \\
\hline Cancer & 13.396 & 8.736 & -4.660 & -3.052 & -1.608 \\
\hline Cerebro. & 2.737 & 1.409 & -1.328 & -0.983 & -0.345 \\
\hline Circulatory & 3.313 & 1.597 & -1.716 & -1.348 & -0.369 \\
\hline Digestive & 2.593 & 2.348 & -0.244 & -0.214 & -0.030 \\
\hline IHD & 13.973 & 5.354 & -8.618 & -7.325 & -1.293 \\
\hline Respiratory & 0.432 & 0.845 & 0.413 & 0.544 & -0.131 \\
\hline Rest & 6.194 & 5.167 & -1.027 & -0.550 & -0.477 \\
\hline$\sum$ & 42.638 & 25.457 & -17.181 & -12.929 & -4.252 \\
\hline \multicolumn{6}{|l|}{ Low education } \\
\hline Cancer & 26.674 & 29.566 & 2.892 & -0.133 & 3.025 \\
\hline Cerebro. & 6.489 & 7.526 & 1.037 & 0.203 & 0.834 \\
\hline Circulatory & 9.668 & 6.553 & -3.115 & -4.144 & 1.029 \\
\hline Digestive & 12.368 & 13.362 & 0.994 & 0.285 & 0.710 \\
\hline IHD & 18.182 & 17.381 & -0.800 & -2.899 & 2.098 \\
\hline Respiratory & 4.072 & 3.507 & -0.565 & -1.102 & 0.537 \\
\hline Rest & 22.863 & 19.301 & -3.562 & -4.345 & 0.783 \\
\hline$\sum$ & 100.315 & 97.196 & -3.118 & -12.135 & 9.016 \\
\hline Cause of death & $\begin{array}{l}\text { High - Low } \\
1981 / 1982\end{array}$ & $\begin{array}{l}\text { High - Low } \\
1991 / 1992\end{array}$ & $\Delta$ & Direct & Comp. \\
\hline
\end{tabular}

High minus low education

\begin{tabular}{lrrrrr} 
Cancer & -13.277 & -20.830 & -7.553 & -2.919 & -4.633 \\
Cerebro. & -3.752 & -6.117 & -2.365 & -1.186 & -1.179 \\
Circulatory & -6.355 & -4.956 & 1.399 & 2.796 & -1.397 \\
Digestive & -9.775 & -11.014 & -1.239 & -0.499 & -0.740 \\
IHD & -4.209 & -12.027 & -7.818 & -4.427 & -3.391 \\
Respiratory & -3.640 & -2.662 & 0.978 & 1.646 & -0.668 \\
Rest & -16.669 & -14.135 & 2.535 & 3.794 & -1.260 \\
$\sum$ & -57.677 & -71.740 & -14.063 & -0.794 & -13.268 \\
\hline
\end{tabular}

disease $(-7.325)$ and cancer (-3.052). For men with low education (middle of Table 3), the observed death rate dropped from 100.315 to 97.196 . This is the outcome of antagonistic developments for the actual improvements in mortality ("Direct") and the changing composition of the population ("Comp."). If the population structure had remained the same for men with low education, mortality would have dropped almost as much for them as it did for men with high education $(-12.135$ vs. -12.929$)$; the compositional component counteracted this development (9.016) which can be traced back to an aging subpopulation of men with low 
Table 4 Decomposition of death rates (per 10,000) of women into direct and compositional ("Comp.") components

\begin{tabular}{|c|c|c|c|c|c|}
\hline Cause of death & $\begin{array}{l}\text { Death rate } \\
1981 / 1982\end{array}$ & $\begin{array}{l}\text { Death rate } \\
1991 / 1992\end{array}$ & $\Delta$ & Direct & Comp. \\
\hline \multicolumn{6}{|l|}{ High education } \\
\hline Cancer & 12.045 & 9.848 & -2.197 & 1.396 & -3.592 \\
\hline Cerebro. & 1.882 & 0.953 & -0.929 & -0.251 & -0.678 \\
\hline Circulatory & 3.388 & 1.271 & -2.117 & -1.159 & -0.958 \\
\hline Digestive & 2.635 & 0.477 & -2.158 & -1.687 & -0.471 \\
\hline IHD & 2.258 & 0.477 & -1.782 & -1.410 & -0.372 \\
\hline Respiratory & 0.376 & 0.635 & 0.259 & 0.646 & -0.387 \\
\hline Rest & 3.011 & 2.859 & -0.152 & -0.153 & 0.001 \\
\hline$\sum$ & 25.596 & 16.520 & -9.076 & -2.618 & -6.457 \\
\hline \multicolumn{6}{|l|}{ Low education } \\
\hline Cancer & 18.603 & 17.834 & -0.769 & -1.375 & 0.606 \\
\hline Cerebro. & 3.824 & 4.051 & 0.227 & -0.040 & 0.266 \\
\hline Circulatory & 5.377 & 4.036 & -1.341 & -1.608 & 0.267 \\
\hline Digestive & 3.624 & 3.464 & -0.160 & -0.220 & 0.060 \\
\hline IHD & 5.142 & 5.711 & 0.569 & 0.146 & 0.423 \\
\hline Respiratory & 1.294 & 1.116 & -0.178 & -0.233 & 0.055 \\
\hline Rest & 8.390 & 5.940 & -2.450 & -2.655 & 0.206 \\
\hline$\sum$ & 46.255 & 42.153 & -4.102 & -5.985 & 1.884 \\
\hline Cause of death & $\begin{array}{l}\text { High - Low } \\
1981 / 1982\end{array}$ & $\begin{array}{l}\text { High - Low } \\
1991 / 1992\end{array}$ & $\Delta$ & Direct & Comp. \\
\hline
\end{tabular}

High minus low education

\begin{tabular}{lrrrrr} 
Cancer & -6.558 & -7.986 & -1.428 & 2.770 & -4.198 \\
Cerebro. & -1.942 & -3.098 & -1.155 & -0.211 & -0.944 \\
Circulatory & -1.990 & -2.766 & -0.776 & 0.449 & -1.225 \\
Digestive & -0.989 & -2.987 & -1.998 & -1.467 & -0.531 \\
IHD & -2.884 & -5.235 & -2.351 & -1.556 & -0.795 \\
Respiratory & -0.918 & -0.481 & 0.437 & 0.880 & -0.443 \\
Rest & -5.378 & -3.081 & 2.298 & 2.502 & -0.205 \\
$\sum$ & -20.659 & -25.633 & -4.974 & 3.367 & -8.341 \\
\hline
\end{tabular}

education. ${ }^{6}$ The largest mortality improvements were found for "all other circulatory diseases" (-4.144), "rest" $(-4.345)$, and ischaemic heart disease $(-2.899)$. The main interest of our analysis is shown in the lower third of Table 3. The gap between men with high education and men with low education increased from 57.677 to 71.740 . The major reason is the changing composition of the population $(-13.268)$. The relatively low impact of direct effects $(-0.794)$ is the

\footnotetext{
${ }^{6}$ Again, we calculated the average age of the population for both time-points. In 1981/1982 the mean age of men with low education was 49.01 years; 10 years later, the corresponding figure was 49.90 years.
} 
result of developments for some causes of death which would have increased the gap even further, most notably ischaemic heart disease $(-4.427)$ and cancer (2.919), and of developments for some causes which would have decreased the differential mortality by education over time: circulatory diseases (2.796), respiratory diseases (1.646) and "rest" (3.794).

As shown in Table 4, observed mortality decreased also for women with high and with low education (High: 25.596 to 16.520; Low: 46.255 to 42.153). Similar to the results for men, we find that compositional changes favored a decrease in mortality for individuals with high education $(-6.457)$, but not for low education (1.884). It is important to note that the direct effect in mortality change was stronger for women with low education $(-5.985)$ than for women with high education (2.618), supporting a narrowing of socioeconomic mortality differentials. This is consequently reflected in the comparison "High minus Low Education" in the lower third of Table 4. The decrease in absolute mortality differentials among women can be attributed to worsening mortality from cancer and respiratory diseases among the highly educated (direct effects are positive: 1.396 and 0.646) and faster decreases in mortality from "All Other Circulatory Diseases" and "Rest" for women with lower education than for women with higher education. Similar to men, we can see that the development of ischaemic heart disease among women would have increased the gradient in socioeconomic mortality differentials (direct effect -1.556) more than any other disease (Digestive Diseases -1.467 , Cerebrovascular Diseases $0.211)$.

\section{Summary}

Our analysis has shown for men that the increasing gap in relative mortality differentials by education were mainly caused by ischaemic heart disease, cerebrovascular diseases and cancers. Only respiratory diseases displayed the opposite trend. Relative mortality differentials from all causes remained almost constant for women. This is the outcome of different developments for various causes of death which cancel each other out on the aggregated level of all causes of death. All circulatory diseases would have increased the gap between women with low and women with high education, whereas respiratory diseases, diseases of the digestive system and the residual category would have diminished educational inequalities in mortality.

The decomposition analysis iterated the finding of relative mortality differentials that ischaemic heart disease and cancer contributed to an increase in socioeconomic mortality differentials for men. We have seen, however, that "all other circulatory diseases", respiratory diseases, and "rest" would have reduced the gap between men of high and of low education. Mortality from ischaemic heart disease contributed to an increasing gap in educational mortality differentials among women as well, together with diseases of the digestive system and cerebrovascular diseases. This development was offset by several other causes of death, most notably cancers. 
Our research has shown one feature which, to our knowledge, has not been reported before: the importance of changes in the population composition for the development of socioeconomic mortality differentials over time. For example, the increasing educational gradient in absolute mortality for men was, on the one hand, the result of direct mortality improvements; the compositional effect was, on the other hand, however, 16 times stronger. ${ }^{7}$ Also within one educational group, compositional effects often play the lead role. One can see, for instance, that deaths from cancer per 10,000 for women with high education dropped from 12.045 to 9.848. This decrease in the observed death rates was, however, not influenced by improvements in mortality. On the contrary, mortality actually increased (1.396) and was offset by a younger population structure as measured by the compositional component (-3.592).

\section{Discussion}

Changes in demographic parameters which can be interpreted as averages can be explained by three broad categories (Vaupel and Canudas-Romo 2002): problems with the data (Level-0), real changes in the phenomenon of interest (Level-1) and compositional changes (Level-2). Hence, we need to discuss first whether data problems or compositional distortions could have an impact before we interpret our results. We exclude poor data as a potential reason for our results because both sources of data, census data and official death registration, are of high quality. Since we were not able to link all death certificates-but more than $90 \%$-with the census, our results for absolute mortality are slightly too low (exact number of exposures but not all events). Although the data did not allow us to check for a potential linkage bias by education, ${ }^{8}$ we assumed that our results are not affected for several reasons: first, our main interest is the development in mortality differentials over time. Even if a linkage bias by education is present, it is rather unlikely that it will change over time. Second, Schwarz (2005) reports for his analysis of Austrian data a decreasing success rate of linked cases with increasing age, ${ }^{9}$ he does not mention a bias in linkage by education, though. Third, a similar data linkage problem was experienced by Huisman et al. (2005a) for Madrid. They describe that "[t]here was no variation by education in the percentage of linkage obtained in this population" (Huisman et al. 2005a, p. 493).

Changes in the composition of a population can have distorting effects for the actual demographic phenomenon of interest. Decomposition analysis enabled us to clearly distinguish between direct effects of developments in mortality and changes

\footnotetext{
${ }^{7}$ As one can see in Table 3, the gap in mortality increased ( -14.063$)$. The contribution of direct mortality improvements $(-0.794)$ was very modest when compared to the compositional effect $(-13.268)$.

${ }^{8}$ Checking for a bias by sex, age, or location was straightforward-see Sect. 2.1.1—as these variables are included not only in the census information but also in the death certificates. Since information on education is not available in the death records, we were unable to compare the distributions of linked and unlinked cases.

${ }^{9}$ His analysis includes ages until 74 .
} 
in the composition of the population. Our analysis has shown that these compositional effects can overshadow direct effects. Regardless of sex, the compositional effect for people with high education favored decreasing mortality whereas the composition of the population of people with low education changed unfavorably for survival. We interpret these effects to be the outcome of the educational expansion in Austria during the 1970s, with more people increasingly attending higher education than in the past. During the first period 1981/1982, this did not yet affect our age-range of 35-64 years. Ten years later, however, the average age of highly educated people decreased due to the replacement effect of the education expansion, while less people "entered" the population with low education causing an aging of this group.

The general picture we have obtained for direct mortality effects has also been found in other countries: cardiovascular diseases and, in particular among them, ischaemic heart disease decreased faster for higher than for lower socioeconomic groups (e.g., Mackenbach et al. 2003; Martikainen et al. 2001; Middelkoop et al. 2001; Steenland et al. 2004; Valkonen et al. 2000). The opposite effect for cancer among women has been documented for several countries (Mackenbach et al. 1999) as well as for The Hague in the Netherlands (Middelkoop et al. 2001).

Especially with the decrease in cardiovascular mortality, Austria shares a "common background" (Koskinen 2003) with other Western European countries, a development which has even been labeled the "cardiovascular revolution" (Meslé and Vallin 2006; Vallin and Meslé 2001). Higher survival for a specific cause of death can be either explained by introducing better technology for treating the disease or by behavioral factors aiming at reducing cardiovascular risk factors. If the first strain of causality was right, the differential pace in cardiovascular mortality improvements could be explained by better access to medical technology for people with higher socioeconomic status than for women and men from lower socioeconomic groups. From an institutional perspective, this is rather unlikely (Bennett et al. 1993; Federal Ministry of Health and Women 2005; Schneider et al. 1995). The Austrian health system is based on the model of compulsory health insurance and hospital care with a maximum additional payment of less than 130 Euros at that time. ${ }^{10}$ "In addition, although private health insurance policies allow patients to choose hospital rooms with slightly more amenities than those for patients covered by the national health system, Austrian laws guarantee that medical care must be similar between the two systems of care" (Bennett et al. 1993). In practice, total equality is not even achieved in the egalitarian Austrian health care system: as a recent study has shown for the OECD countries (van Doorslaer et al. 2006), there is almost no socio-economic gradient in the probability of visiting a doctor in general; richer people, however, are more likely to see a specialist. This might have some influence. We assume, nevertheless, that these reductions in cardiovascular mortality are the outcome of changes in risk-factor related behavior-a point of

\footnotetext{
${ }^{10}$ According to Schneider et al. (1995, p. 391), this maximum was due in the case of 28 or more days as an inpatient in a hospital. It should be additionally mentioned that even in countries like the United States, which are less egalitarian than Austria, differences in socioeconomic groups turned out to be rather negligible in the analysis of "who is at greatest risk for receiving poor-quality health care?" as a recent study has shown (Asch et al. 2006).
} 
view which is also typically expressed in the literature (e.g., Breslow 1985). Another example is the study of all-cause mortality, cardiovascular mortality, and acute myocardial infarction by Lynch et al. (1996). They state that their "findings imply that efforts to reduce the disease burden associated with low SES can legitimately focus on the behavior, psychosocial and biologic risk factors that mediate the relation" (p. 941).

In a recently published working paper, Schwarz (2006) illustrates that the typical behavioral risk factors such as healthy diet, physical exercise and obesity have a social gradient in Austria. Other studies for Austria also point in this direction. Schmeisser-Rieder and Kunze (2000), for example, have shown that during their investigation which spanned the years 1978-1998 a declince was observed in the proportion of people in Austria who knew their blood pressure and of people who had their blood pressure measured during the last 3 months before the interview. This could turn out to be problematic since women and men with lower education tend to have higher blood pressure (de Gaudemaris et al. 2002). Obesity, another risk factor for ischaemic heart disease, also shows a social gradient in Austria as demonstrated by Kirchengast et al. (2004). The major risk factor for all cardiovascular diseases and many other diseases is smoking. During our observation period, the prevalence of smoking increased-especially for women (Haidinger et al. 1998; World Health Organization 2006). Studies for our age-range found out that a social gradient for smoking almost does not exist for women in Austria (Huisman et al. 2005b). As shown by Schwarz (2006), there is a crossover by social gradient among women. Before the age of 45 , lower educated women tend to smoke more, whereas smoking is more prevalent among higher educated women at later ages. This crossover could also explain the differential development for cancer and ischemic heart disease among women. If smoking has an impact on both causes of death but for cancer at a different age than for ischaemic heart disease, it is possible that cancer rather diminished the gap in socioeconomic mortality differentials whereas ischaemic heart disease increased it.

\section{Conclusions}

The article has shown that socioeconomic inequalities in mortality persisted or even increased for people at working ages in Austria for all causes of death between 1981/1982 and 1991/1992. Our analysis presented the causes of death which showed a clearly widening gap in socioeconomic mortality differentials. The identification of such vulnerable population subgroups is only the first step and probably the easiest step for reducing inequalities in mortality which has been one of the major targets of the World Health Organization Europe since the 1980s. In the 2002 European Health Report, the first goal mentioned is "reducing excess mortality, morbidity and disability, especially in poor and marginalized populations" (WHO Regional Office for Europe 2002, p. 2). Implementing effective policies is, however, not straightforward. Policies which try to tackle these inequalities will face various stumbling-blocks. First, at which age should public health policies start? Besides the aforementioned risk factors, a growing amount 
of literature suggests that the onset of many diseases, and in particular cardiovascular diseases, are affected by early childhood or even fetal life (e.g., Barker 1995; Doblhammer 2004; Elo and Preston 1992; Eriksson 2005). Second, consequences of current public health policies may be hard to assess due to lagged effects. As Lopez et al. (1994, p. 243) diagnoses: "death rates from lung cancer generally do not reach their maximum until 30 or 40 years after the peak in smoking prevalence." Third, one can doubt whether public health policies are successful at all in reducing levels of inequality. In a recent study, socioeconomic mortality differentials turned out to be larger in the rather egalitarian country of Denmark than in the United States, which is typically labeled to be rather a liberal, laissez-faire country (Hoffmann 2006). Pamuk (1985) found increasing levels in inequality in mortality in England and Wales after the introduction of the National Health Service in 1948. Also Preston and Elo (1995) found increasing socioeconomic inequality in mortality in the United States between 1960 and 1985-despite the introduction of Medicare in 1966. ${ }^{11}$ Finally, it should be added that the assessment of whether socioeconomic differences in mortality are changing over time within a country is becoming more challenging. Changes in the size and composition of socioeconomic groups can be accounted for, as we have done in this study. The meaning of belonging to a certain group at a certain point of time can change dramatically, however, for instance, attending school which is classified as "low education" used to be the norm in many countries for long periods of time, whereas nowadays pupils with basic schooling are rather from a selected population. This problem applies equally to many occupational groups. Widening gaps in socioeconomic mortality differentials can, therefore, also be the outcome of stronger selection over time into a specific socioeconomic category.

Acknowledgments We would like to thank Josef Kytir from Statistics Austria for providing the data. Some parts and earlier versions of this article have been previously presented at an RTN Workshop in Sardinia (2005), Warsaw School of Economics (2005), PAA Annual Meeting in Los Angeles (2006), the "Rostock Demographic Colloquium" (2006) and the European Population Conference in Liverpool (2006). We would like to thank for all the comments and suggestions we received, especially from James W. Vaupel, Vladimir Shkolnikov, and the anonymous reviewers of this article who improved our analysis and this article enormously.

\section{References}

Asch, S. M., Kerr, E. A., Keesey, J., Adams, J. L., Setodji, C. M., Malik, S., \& McGlynn, E. A. (2006). Who is at greatest risk for receiving poor-quality health care? New England Journal of Medicine, $354,1147-1156$.

Barker, D. (1995). Fetal origins of coronary heart disease. British Medical Journal, 311, 171-174.

Bennett, C. L., Schwarz, B., \& Marberger, M. (1993). Health care in Austria: Universal access, national health insurance, and private health care. Journal of the American Medical Association, 269, 27892794.

\footnotetext{
11 One can, of course, argue that "[t]hese findings do not imply that egalitarian socio-economic policies cannot help to reduce socio-economic differences in mortality. It is more than likely that mortality differences in the Nordic countries would have been larger in the absence of egalitarian policies, or that mortality differences in the United States would have been smaller if income inequalities in this country would have been as small as in Nordic countries." (Kunst 1997, p. 142).
} 
Breslow, L. (1985). The case of cardiovascular diseases. In J. Vallin \& A. D. Lopez (Eds.), Health policy, social policy and mortality prospects (Chapter 9, pp. 197-216). Paris, France: INED \& IUSSP.

Canudas-Romo, V. (2003). Decomposition methods in demography. Amsterdam, Netherlands: Rozenberg Publishers.

Christenson, B. A., \& Johnson, N. E. (1995). Educational inequality in adult mortality: An assessment with death certificate data from Michigan. Demography, 32, 215-229.

Das Gupta, P. (1994). Standardization and decomposition of rates from cross-classified data. Genus, 50, 171-196.

Davey Smith, G., Bartley, M., \& Blane, D. (1990). The Black report on socioeconomic inequalities in health 10 years on. British Medical Journal, 301, 373-377.

Davey Smith, G., Hart, C., Hole, D., MacKinnon, P., Gillis, C., Watt, G., Blane, D., \& Hawthorne, V. (1998). Education and occupational social class: Which is the more important indicator of mortality risk? Journal of Epidemiology and Community Health, 52, 153-160.

de Gaudemaris, R., Lang, T., Chatelliert, G., Larabi, L., Lauwers-Cancès, V., Maître, A., \& Diène, E. (2002). Socioeconomic inequalities in hypertension prevalence and care: The IHPAF study. Hypertension, 39, 1119-1125.

Doblhammer, G. (1997). Socioeconomic differentials in Austrian adult mortality. PhD thesis, Sozial- und Wirtschaftswissenschaftliche Fakultät, Universität Wien, Vienna, Austria.

Doblhammer, G. (2004). The late life legacy of very early life. Demographic Research Monographs. Springer, Heidelberg, Germany.

Doblhammer, G., Rau, R., \& Kytir, J. (2005). Trends in educational and occupational differentials in allcause mortality in Austria between 1981/82 and 1991/92. Wiener Klinische Wochenschrift, 117, $468-479$.

Elo, I. T., Martikainen, P., \& Smith, K. P. (2006). Socioeconomic differentials in mortality in Finland and the United States: The role of education and income. Europen Journal of Population, 22, 179-203.

Elo, I. T., \& Preston, S. H. (1992). Effects of early-life conditions on adult mortality: A review. Population Index, 58, 186-212.

Eriksson, J. G. (2005). The fetal origins hypothesis-10 years on. British Medical Journal, 330, 10961097.

Federal Ministry of Health and Women (2005). Public health in Austria (4th updated ed.). Vienna, Austria: Federal Ministry of Health and Women.

Feldman, J. J., Makuc, D. M., Kleinman, J. C., \& Cornoni-Huntley, J. (1989). National trends in educational differentials in mortality. American Journal of Epidemiology, 129, 919-933.

Goldman, N. (2001). Mortality differentials: Selection and causation. In N. J. Smelser \& P. B. Baltes (Eds.), International encyclopedia of the social \& behavioral sciences (pp. 10068-10070) Amsterdam, Netherlands: Elsevier.

Haidinger, G., Waldhoer, T., \& Vutuc, C. (1998). The prevalence of smoking in Austria. Preventive Medicine, 27, 50-55.

Hoffmann, R. (2006). Socioeconomic differences in old age mortality in Denmark and the USA with special emphasis on the impact of unobserved heterogeneity on the change of mortality differences over age. PhD thesis, Universität Rostock, Rostock, Germany.

Huisman, M., Kunst, A. E., Bopp, M., Borgan, J.-K., Borrell, C., Costa, G., Deboosere, P., Gadeyne, S., Glickman, M., Marinacci, C., Minder, C., Regidor, E., Valkonen, T., \& Mackenbach, J. P. (2005a). Educational inequalities in cause-specific mortality in middle-aged and older men and women in eight western european populations. The Lancet, 365, 493-500.

Huisman, M., Kunst, A. E., \& Mackenbach, J. P. (2005b). Inequalities in the prevalence of smoking in the European Union: Comparing education and income. Preventive Medicine, 40, 756-764.

Kirchengast, S., Schober, E., Waldhoer, T., \& Sefranek, R. (2004). Regional and social differences in body mass index, and the prevalence of overweight and obesity among 18 year old men in Austria between the years 1985 and 2000. Collegium Antropologicum, 28, 541-552.

Kitagawa, E. M. (1955). Components of a difference between two rates. Journal of the American Statistical Association, 50, 1168-1194.

Kitagawa, E. M., \& Hauser, P. M. (1973). Differential mortality in the United States: A study in socioeconomic epidemiology. Cambridge, MA: Harvard University Press.

Koskinen, S. (2003). Commentary: Is there a common background behind growing inequalities in mortality in Western European countries? International Journal of Epidemiology, 32, 838-839.

Kunst, A. (1997). Cross-national comparisons of socio-economic differences in mortality. PhD thesis, Department of Public Health, Erasmus University Rotterdam, Rotterdam, Netherlands. 
Kunst, A. E., Bos, V., Andersen, O., Cardano, M., Costa, G., Harding, S., Hemström, O., Layte, R., Regidor, E., Reid, A., Santana, P., Valkonen, T., \& Mackenbach, J. P. (2004). Monitoring of trends in socioeconomic inequalities in mortality: Experiences from a European project. Demographic Research, Special Collection, 2, 229-253.

Lauderdale, D. S. (2001). Education and survival: Birth cohort, period, and age effects. Demography, 38, 551-561.

Leinsalu, M., Vågerö, D., \& Kunst, A. E. (2003). Estonia 1989-2000: Enourmous increase in mortality differentials by education. International Journal of Epidemiology, 32, 1081-1087.

Liberatos, P., Link, B. G., \& Kelsey, J. L. (1988). The measurement of social class in epidemiology. Epidemiologic Reviews, 10, 87-121.

Lopez, A. D., Collishaw, N. E., \& Piha, T. (1994). A descriptive model of the cigarette epidemic in developed countries. Tobacco Control, 3, 242-247.

Lynch, J. W., Kaplan, G. A., Cohen, R. D., Tuomilehto, J., \& Salonen, J. T. (1996). Do cardiovascular risk factors explain the relation between socioeconomic status, risk of all-cause mortality, cardiovascular mortality, and acute myocardial infarction? American Journal of Epidemiology, 144, 934-942.

Lynch, J. W., Kaplan, G. A., \& Salonen, J. T. (1997). Why do poor people behave poorly? Variation in adult health behaviors and psychosocial characteristics by stages of the socioeconomic lifecourse. Social Science and Medicine, 44, 809-819.

Mackenbach, J. P., Bos, V., Andersen, O., Cardano, M., Costa, G., Harding, S., Reid, A., Hemström, Ö., Valkonen, T., \& Kunst, A. E. (2003). Widening socioeconomic inequalities in mortality in six Western European countries. International Journal of Epidemiology, 32, 830-837.

Mackenbach, J. P., \& Kunst, A. E. (1997). Measuring the magnitude of socio-economic inequalities in health: An overview of available measures illustrated with two examples from Europe. Social Science \& Medicine, 44, 757-771.

Mackenbach, J. P., Kunst, A. E., Groenhof, F., Borgan, J.-K., Costa, G., Faggiano, F., Józan, P., Leinsalu, M., Martikainen, P., Rychtarikova, J., \& Valkonen, T. (1999). Socioeconomic inequalities in mortality among women and among men: An international study. American Journal of Public Health, 89, 1800-1806.

Marmot, M. (2005). Social determinants of health inequalities. The Lancet, 365, 1099-1104.

Martikainen, P., Valkonen, T., \& Martelin, T. (2001). Change in male and female life expectancy by social class: Decomposition by age and cause of death in Finland 1971-95. Journal of Epidemiology and Community Health, 55, 494-499.

McCullagh, P., \& Nelder, J. (1989). Generalized linear models. London, UK: Chapman and Hall.

Meslé, F., \& Vallin, J. (1996). Reconstucting long-term series of causes of death. The case of France. Historical Methods, 29, 72-87.

Meslé, F., \& Vallin, J. (2006). The health transition: Trends and prospects. In G. Caselli, J. Vallin, \& G. Wunsch (Eds.), Demography. Analysis and synthesis (Vol. II, Chapter 57, pp. 247-259). Amsterdam Netherlands: Elsevier.

Middelkoop, B. J., Struben, H. W., Burger, I., \& Vroom-Jongerden, J. M. (2001). Urban cause-specific socioeconomic mortality differences. Which causes of death contribute most? International Journal of Epidemiology, 30, 240-247.

Pamuk, E. R. (1985). Social class inequality in mortality from 1921 to 1972 in England and Wales. Population Studies, 39, 17-31.

Pappas, G., Queen, S., Hadden, W., \& Fisher, G. (1993). The increasing disparity in mortality between socioeconomic groups in the United States, 1960 and 1986. The New England Journal of Medicine, 329, 103-109.

Preston, S. E., \& Elo, I. T. (1995). Are educational differentials in adult mortality increasing in the United States? Journal of Aging and Health, 7, 476-496.

Schmeisser-Rieder, A., \& Kunze, U. (2000). Blood pressure awareness in Austria. A 20-year evaluation, 1978-1998. European Heart Journal, 21, 414-420.

Schneider, M., Biene-Dietrich, P., Gabanyi, M., Hofmann, U., Huber, M., Aynur, K., \& Sommer, J. H. (1995). Gesundheitssysteme im international Vergleich. Ausgabe 1994. Laufende Berichterstattung zu ausländischen Gesundheitssystemen. Augsburg, Germany: BASYS.

Schwarz, F. (2005). Widening educational inequalities in mortality: An analysis for Austria with international comparisons. Working Paper 07/2005, Vienna Institute of Demography. Austrian Academy of Sciences, Vienna, Austria. 
Schwarz, F. (2006). Behavioral explanation for educational health and mortality differentials in Austria. Working Paper 03/2006, Vienna Institute of Demography. Austrian Academy of Sciences, Vienna, Austria.

Shkolnikov, V., Meslé, F., \& Vallin, J. (1996). Health crisis in Russia I. Recent trends in life expectancy and causes of death from 1970 to 1993. Population. An English Selection, 8, 123-154.

Smith, J. P. (1999). Healthy bodies and thick wallets: The dual relation between health and economic status. The Journal of Economic Perspectives, 13, 145-166.

Smith, J. P. (2005). The impact of SES on health over the life-course. Working Paper WR-318. RAND Corporation, Santa Monica, CA.

Steenland, K., Hu, S., \& Walker, J. (2004). All-cause and cause-specific mortality by socioeconomic status among employed persons in 27 US States, 1984-1997. American Journal of Public Health, 94, 1037-1042.

Valkonen, T. (1989). Adult mortality and level of education: A comparison of six countries. In A. Fox (Ed.), Health inequalities in European countries (pp. 124-162). Aldershot, UK: Gower.

Valkonen, T. (1999). The widening differentials in adult mortality by socio-economic status and their causes. In United Nations (Ed.), Health and mortality. Issues of global concern (pp. 291-312). New York, NY: United Nations.

Valkonen, T. (2006). Social inequalities in mortality. In G. Caselli, J. Vallin, \& G. Wunsch (Eds.), Demography. Analysis and synthesis (Vol. II, Chapter 54, pp. 195-206). Amsterdam, Netherlands: Elsevier.

Valkonen, T., Martikainen, P., Jalovaara, M., Koskinen, S., Martelin, T., \& Mäkelä, P. (2000). Changes in socioeconomic inequalities in mortality during an economic boom and recession among middleaged men and women in Finland. European Journal of Public Health, 10, 274-280.

Vallin, J., \& Meslé, F. (2001). Trends in mortality in Europe since 1950: Age-, sex- and cause-specific mortality. In J. Vallin, F. Meslé, \& T. Valkonen (Eds.) Trends in mortality and differential mortality. Population studies (Vol. 36, pp. 31-184). Strasbourg, France: Council of Europe Publishing.

van Doorslaer, E., Masseria, C., \& Koolman, X. (2006). Inequalities in access to medical care by income in developed countries. Canadian Medical Association Journal, 174, 177-183.

Vaupel, J. W., \& Canudas-Romo, V. (2002). Decomposing demographic change into direct vs. compositional components. Demographic Research, 7, 1-14.

Vaupel, J. W., \& Canudas-Romo, V. (2003). Decomposing change in life expectancy: A bouquet of formulas in Honor of Nathan Keyfitz's 90th birthday. Demography, 40, 201-216.

Vaupel, J. W., Canudas-Romo, V., \& Zhang, Z. (2006). Analysis of population changes and differences. Book Manuscript, Max Planck Institute for Demographic Research, Rostock, Germany, Konrad Zuse Str. 1, D-18057 Rostock, Germany.

WHO Regional Office for Europe (2002). The European Health Report 2002. WHO Regional Publications, European Series 97, World Health Organization, Regional Office for Europe, Copenhagen, Denmark.

Woodward, M. (1999). Epidemiology. Study design and data analysis. Boca Raton, FL: Chapman and Hall.

World Health Organization (2006). Global status report - Austria - 1997. Available online at: http://www.cdc.gov/tobacco/WHO/austria.htm 